\title{
Importancia de las competencias instrumentales en formación de docentes para educación secundaria
}

\author{
Importance of instrumental skills in the training of secundary educational \\ teachers
}

\author{
Minerva Atenea Ojeda, Margarita Rosa Pino-Juste, Jorge Soto Carballo \\ Universidad de Vigo
}

\begin{abstract}
Resumen
Uno de los criterios utilizados para evaluar programas de educación superior consiste en conocer la importancia que los egresados otorgan a las diferentes competencias planteadas en los contenidos curriculares. El objetivo de este trabajo es determinar el grado de importancia que los egresados del Master en educación secundaria de la Universidad de Vigo dan a un conjunto de competencias instrumentales, presentando los resultados de un cuestionario aplicado a una muestra representativa. Las competencias instrumentales evaluadas se formularon a partir de los contenidos curriculares de dicho Master y comprenden habilidades cognitivas, capacidades metodológicas, destrezas tecnológicas y habilidades lingüísticas.

Palabras clave: competencias del docente, formación de docentes, evaluación de la educación.
\end{abstract}

\section{Abstract}

One of the criteria used to evaluate higher education programs is to know the importance that the graduates give to the different competencies raised in the curricular contents. The objective of this work is to determine the importance level that the graduates of the Master in secondary education of the University of Vigo give to a set of instrumental competences, presenting the results of a questionnaire applied to a representative sample. The instrumental competences evaluated were formulated from the curricular contents of the Master and include cognitive abilities, methodological capacities, technological skills and linguistic skills.

Key words: teacher qualification, teacher education, educational evaluation.

La estrategia europea "Educación y Formación 2020" aprobada por el Consejo de Europa, 12 de mayo de 2009, presta especial atención al papel del profesorado, desde su elección, formación inicial y desarrollo profesional continuo, a sus oportunidades profesionales (European Union, 2009). Esto conlleva que se debe seleccionar concienzudamente al profesorado, proporcionarle una formación eficaz y adecuada, retenerlo en su puesto, brindarle apoyo en las primeras fases de su carrera profesional y ofrecerle regularmente oportunidades para poner al día sus aptitudes y competencias, incluyendo la adopción de nuevas tecnologías (European Comission/EACEA/Eurydice, 2015). En junio de 2015 se han publicado dos informes técnicos sobre las prácticas docentes del profesorado en la unión europea. Como señalan De Juanas Oliva y Beltrán Llera (2014) este escenario social impulsó el proceso de cambio en el que se encuentra la educación superior europea tras la Declaración de Bolonia.

Los conocimientos, capacidades y aptitudes de los seis millones de profesores europeos son importantísimos ya que la calidad de su enseñanza influye directamente en los resultados académicos de su alumnado (European Comission, 2013).

Por ello señalan Aparicio \& Moneo (2016) que "existe un acuerdo general entre los economistas de la educación respecto a que uno de los principales motores del progreso social y económico de un país es el llamado capital humano".

Dada la importancia del tema en este estudio se pretende describir la opinión de los graduados en el master de secundaria sobre el dominio de competencias instrumentales. Para ello comenzamos por acercarnos al concepto utilizado. Consideramos importante distinguir entre las competencias docentes (teaching competences) y las competencias del profesorado (teacher competences) (OCDE, 2009). Entendemos con Hagger y McIntyre (2006) por competencias docentes aquellas que se centran en el papel del profesor en el aula, directamente vinculados al "oficio" de la enseñanza - con conocimientos y habilidades profesionales movilizados para la acción, mientras que las competencias del profesorado implican una visión más amplia y sistemática de la profesionalización del profesorado, en múltiples ámbitos -el individuo, la escuela, la comunidad local y las redes profesionales.

Kelly and Grenfell's European Profile of Language Teacher Education (2004) y el Proyecto Tuning (2005) han desarrollado las pautas sobre las competencias del profesorado y proporcionan marcos de referencia para los programas de formación del profesorado europeo.

La formación inicial del profesorado varía en función de los países de la Unión Europea. El nivel de cualificación de máster (CINE 7) es el nivel mínimo de cualificación para trabajar en el primer ciclo de la 
educación secundaria general en 17 países. Dichos países son: la República Checa, Alemania, Estonia, España, Francia, Croacia, Italia, Luxemburgo, Hungría, Polonia, Portugal, Eslovenia, Eslovaquia, Finlandia, Suecia, Islandia y Serbia. En 15 países solo se requiere el nivel de licenciado (CINE 6). Dichos países son: Bélgica (Comunidades Francesa y Flamenca), Bulgaria, Dinamarca, Irlanda, Grecia, Chipre, Letonia, Lituania, Malta, Rumanía, Reino Unido, Montenegro, la Antigua República Yugoslava de Macedonia, Noruega y Turquía (European Comission (2015).

En España la Ley Orgánica de Educación (2006) describe las competencias del profesorado y su formación inicial está regulada por las directrices descritas en los programas de las diferentes titulaciones universitarias. De todos modos, se está debatiendo sobre el empleo de descriptores de las competencias para la evaluación del desarrollo profesional de los profesores.

\section{Método}

\section{Participantes}

La muestra estuvo conformada por 155 egresados del master de secundaria de la Universidad de Vigo. E1 $31 \%$ hombres mientras que el $69 \%$ son mujeres. La edad media es de 30, 46 siendo el mayor de 49 años y el menor de 22 .

\section{Instrumento}

El instrumento utilizado es el diseñado por Tribó (2008) que pretende evaluar la percepción sobre las competencias profesionales específicas que debe desarrollar un profesor de secundaria. Se agrupan las competencias profesionales específicas en los cuatro ámbitos de competencias: científicas, metodológicas, sociales y personales. En este estudio se analizarán las dos primeras denominadas habitualmente como instrumentales.

La selección de las competencias se ha realizado apoyándose, por un lado, en las aportaciones de publicaciones clásicas como el informe de la UNESCO (1996), el informe Tuning (2003), ya que ambos representan a colectivos importantes de expertos y de profesionales de la educación..

Dentro de las competencias disciplinares o científicas (ámbito del saber) se incluyen los conocimientos teóricos de la disciplina, de la didáctica, de la pedagogíapsicología, de las TIC e idiomas. Dentro de las competencias metodológicas o técnicas (ámbito del saber hacer) se incluyen la gestión del aula, técnicas de trabajo en equipo, atención a la diversidad, resolución de conflictos, programaciones didácticas, evaluación y uso de las TIC.

\section{Procedimiento}

El estudio se llevó a cabo siguiendo las normas deontológicas reconocidas por la Declaración de Helsinki (revisión de Hong-Kong, septiembre de 1989) y de acuerdo con las recomendaciones de Buena Práctica Clínica de la CEE (documento 111/3976/88 de julio de 1990) y la normativa legal vigente española que regula la investigación.
El cuestionario se administró de manera colectiva a los egresados en la universidad de Vigo mediante un cuestionario en línea durante el curso 2016-17.

\section{Análisis de los datos}

Se llevó a cabo, en primer lugar, un análisis descriptivo de los ítems, porcentajes, media, desviación típica, así como los índices de asimetría y curtosis para evaluar el comportamiento normal de las variables. Los resultados mostraron un coeficiente alfa de $\alpha=.96$ para la escala total, subescala competencias científicas $\alpha=.915$, subescala competencias metodológicas $\alpha=.952$ lo que implica una alta fiabilidad en ambos casos (George \& Mallery, 2003; Gliem \& Gliem, 2003; Sijtsma, 2009).

A continuación, se emplea la prueba de Kolmogorov Smirnov con el propósito de decidir de un modo riguroso, si la muestra de la que se dispone procede o no de una distribución normal $\mathrm{y}$, seguidamente, se realizaron pruebas $\mathrm{t} y$ ANOVA de comparación de medias independientes con un nivel de significación de $\mathrm{p}<.05$.

Se ha utilizado un nivel de confianza de.05

Para el análisis de los datos se emplearon el programa estadístico SPSS 23.0.

\section{Resultados}

La mayoría de los egresados están solteros (82.6\%) y esto puede ser debido a que se ha seleccionado a los egresados de los últimos cinco años.

El reparto muestral por ámbitos de conocimiento ha sido muy equilibrado. El $14.8 \%$ ha realizado sus estudios en grados del ámbito científico (Física, Química, ciencias del mar enfermería o biología), el $37.3 \%$ dentro del ámbito de las Humanidades (distintas filologías, traducción e interpretación, historia, Bellas Artes y filosofía), el $26.1 \%$ dentro del ámbito de las ciencias sociales y jurídicas (psicopedagogía, pedagogía, ciencias de la actividad física y deporte, magisterio, economía, derecho, dirección y administración de empresas) y el 21 . $6 \%$ en el ámbito tecnológico (arquitectura y las diferentes ingenierías).

La mayoría de los egresados del master de secundaria no trabaja en la enseñanza (61. 3\%) aunque algunos de ellos trabajan impartiendo clases particulares, pero muchos de camareros, comerciales o empleos relacionados con la administración.

Llama la atención el hecho de que apenas un 51\% de los encuestados considera importante hablar una lengua extranjera, en este caso, el inglés; aunque es la competencia más valorada, seguida de tener un alto dominio de las lenguas oficiales (41.9\%). Siendo la de conocer estrategias educativas innovadoras $(9,7 \%)$ a la que menos importancia se le da, seguida de saber planificar y organizar los contenidos, con la valoración de apenas un $10.3 \%$ de la población.

Con relación al dominio de las TIC para su uso como recurso en clase, un porcentaje muy bajo de los egresados la consideran una competencia importante para su desempeño como docentes, apenas un $13.5 \%$. Por otro lado, un $31 \%$ de los egresados, considera muy importante saber resolver los conflictos que se presentan dentro del aula, sin embargo, un $44.5 \%$ considera que no es importante aplicar estrategias para que los alumnos 
trabajen en equipo o colaboración. Así como un 39.2\% de los egresados que consideran irrelevante saber gestionar el clima del aula (ambiente de trabajo, confianza, diálogo).

Tabla 1.

Porcentajes de respuesta de cada ítem

\begin{tabular}{lllll}
\hline COMPETENCIAS & 0 & 1 & 2 & 3 \\
\cline { 2 - 4 } COMPETENCIAS & Porcentajes & & \\
CIENTÍFICAS (Saber) & & & & \\
\hline
\end{tabular}

1. Conocer los contenidos

de la materia que imparto

2. Estar actualizado sobre $20 \quad 31,6 \quad 35,5 \quad 12,9$

las novedades en la materia

3. Conocer la historia y $27,7 \quad 38,7 \quad 24,5 \quad 9$

evolución de la materia de

la que soy profesor

4. Saber transferir mis conocimientos para que el alumno aprenda

5. Saber planificar y $10,3 \quad 27,7 \quad 42,6 \quad 19,4$ organizar los contenidos

6. Tener formación en pedagogía y psicología aplicada a jóvenes

7. Conocer estrategias educativas innovadoras

8. Conocer las TIC (nuevas tecnologías, Internet)

9. Tener un alto nivel de competencia lingüística en lenguas oficiales

10. Conocer una lengua $51 \quad 26,5 \quad 14,8 \quad 7,7$ extranjera para poder dar clases (inglés)

\section{COMPETENCIAS Porcentajes}

METODOLÓGICAS

(Saber hacer)

1. Gestionar el clima del $21,3 \quad 34,8 \quad 32,9 \quad 11$ aula (ambiente de trabajo, confianza, diálogo...)

2. Aplicar estrategias para que los alumnos trabajen en grupo

3. Fomentar dinámicas para la cohesión de la clase

4. Dar respuesta a los problemas de diversidad en el aula

5. Elaborar propuestas para alumnado con necesidades educativas

6. aber resolver conflictos dentro del aula

7. Desarrollar las tutorías y saber orientar académica y profesionalmente

8. Introducir elementos de mejora, tras una reflexión sobre mi práctica

\begin{tabular}{|c|c|c|c|c|}
\hline $\begin{array}{l}\text { 9.Dominar competencias } \\
\text { comunicativas verbales y } \\
\text { no verbales }\end{array}$ & 21,3 & 34,2 & 31,6 & 12,9 \\
\hline $\begin{array}{l}\text { 10. Utilizar diferentes } \\
\text { técnicas y sistemas de } \\
\text { evaluación del alumnado }\end{array}$ & 12,3 & 27,7 & 41,9 & 18,1 \\
\hline $\begin{array}{l}\text { 11. Explorar conocimiento } \\
\text { previo del } \\
\text { mediante una } \\
\text { inicial }\end{array}$ & 12,3 & 26,5 & 43,9 & 17,4 \\
\hline $\begin{array}{l}\text { 12. Realizar seguimiento } \\
\text { del grado de aprendizaje de } \\
\text { los alumnos }\end{array}$ & 11,6 & 31 & 44,5 & 12,9 \\
\hline $\begin{array}{l}\text { 13. Utilizar la evaluación } \\
\text { como herramienta de } \\
\text { mejora de aprendizaje }\end{array}$ & 15,5 & 27,7 & 41,9 & 14,8 \\
\hline $\begin{array}{l}\text { 14.Diseñar una } \\
\text { programación didáctica }\end{array}$ & 18,7 & 21,3 & 40 & 20 \\
\hline $\begin{array}{l}\text { 15. Saber elaborar } \\
\text { materiales didácticos }\end{array}$ & 14,2 & 36,8 & 34,2 & 14,9 \\
\hline $\begin{array}{l}\text { 16. Dominar las TIC para } \\
\text { utilizarlas como recurso en } \\
\text { clase }\end{array}$ & 14,2 & 31 & 34,8 & 20 \\
\hline $\begin{array}{l}\text { 17. Educar al alumnado } \\
\text { para la información y } \\
\text { comunicación tecnológica }\end{array}$ & 19,4 & 37,4 & 31,6 & 11,6 \\
\hline
\end{tabular}

En la tabla 2, podemos observar que el alumnado tiene la opinión de que posee una formación en competencias científicas y metodológicas más bien bajas.

Tabla 2.

Descriptivos de las competencias

\begin{tabular}{lll}
\hline $\mathrm{N}=155$ & $\begin{array}{c}\text { Competencias } \\
\text { científicas }\end{array}$ & $\begin{array}{c}\text { Competencias } \\
\text { metodológicas }\end{array}$ \\
\hline Media & 1.3942 & 1.3457 \\
Mediana & 1.4000 & 1.2941 \\
Moda & $1.30(\mathrm{a})$ & 1.00 \\
Desv. típ. & .70483 & .67013 \\
Asimetría & .223 & .212 \\
Curtosis & -.503 & -.123 \\
Mínimo & .00 & .00 \\
Máximo & 3.00 & 3.00 \\
\hline
\end{tabular}

En la tabla 3, si agrupamos los resultados, podemos ver que la mayoría del alumnado tiene una percepción media de sus competencias $(50.3 \%)$ y que incluso el $33.5 \%$ opina que tiene bajas competencias científicas. Con respecto a las competencias metodológicas, el $49 \%$ opina que tiene unas competencias medias, pero el $36.1 \%$ piensa que las tiene bajas. 
Tabla 3.

Porcentaje de egresados según nivel de competencias

\begin{tabular}{llcc}
\hline & & & \\
& & Frecuencia & \% válido \\
\hline Competencias & Bajo & 52 & 33.5 \\
científicas & Medio & 78 & 50.3 \\
& Alto & 25 & 16.1 \\
& Total & 155 & 100 \\
\hline Competencias & Bajo & 56 & 36.1 \\
metodológicas & Medio & 76 & 49.0 \\
& Alto & 23 & 14.8 \\
& Total & 155 & 100 \\
\hline
\end{tabular}

Hemos comprobado si existían diferencias entre los grupos en función del ámbito científico en el que se han especializado. Como puede apreciarse en la tabla 3, no existen diferencias sobre la percepción de competencias metodológicas, aunque el alumnado de humanidades se considera más competente. Pero en lo que respecta a la percepción de las competencias científicas si hay diferencias ente las percepciones de los egresados de humanidades e ingeniería, sintiéndose más competentes para la enseñanza los primeros.

Tabla 4.

Diferencia de medias de los diferentes con respecto al ámbito

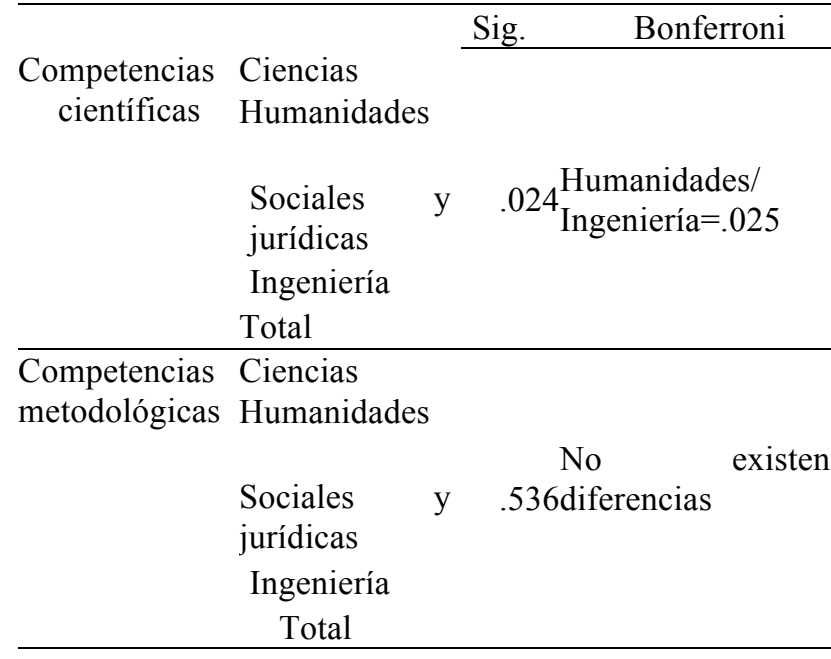

Con relación a la percepción del dominio de competencias por sexo, no se encontró ninguna diferencia significativa; tampoco hay diferencias entre hombres y mujeres con respecto a si trabajan o no en algo relacionado con la enseñanza.

\section{Discusión}

La calidad de la educación de los países depende de la calidad de su profesorado (Galaz, 2015:53), y esta depende a su vez del tipo de formación recibida. Por ello la formación del profesorado resulta un proceso crucial en el ámbito educativo, fundamental para la enseñanza y para el futuro de las nuevas generaciones. De hecho, Mir y Ferrer (2014) señalan que la formación de las personas es clave para el desarrollo de la sociedad actual, ya que esta sociedad del conocimiento debe lograr mantener y mejorar los niveles de bienestar.

Las medidas para evaluar el desarrollo de las competencias de los docentes son importantes porque: no solamente pueden sensibilizar al profesor sobre la necesidad de desarrollar sus competencias, también pueden apoyar una transformación en la enseñanza de la cultura y la práctica y permitir el reconocimiento de las (nuevas) competencias adquiridas o desarrolladas, así como desempeñar un papel en la garantía de la calidad y el control de la formación y el desarrollo. Puede ayudar a desarrollar la confianza en el desempeño docente; Y puede facilitar una intervención oportuna para mejorar la enseñanza (European Comission, 2013).

Lo resultados obtenidos nos llevan a plantearse si la formación que se les da a los estudiantes del master de educación secundaria es suficiente y si se están consiguiendo proyectar las competencias futuras que requerirán para su buen desempeño y poder trabajar dentro del ámbito docente. Esto debido a que los resultados que tienen que ver con la sensación del manejo o dominio de las competencias adquiridas que presentan los egresados del master de educación secundaria de la Universidad de Vigo son un tanto preocupantes, o por lo menos son en contraste con un $50.3 \%$ de los egresados del master en educación secundaria de la Universidad de Vigo que consideran que consideran que tienen un manejo medio de las competencias adquiridas, y con un $36.1 \%$ de estos que considera que el dominio que tiene de las competencias es bajo.

Por otro lado, al haber encontrado que solamente la mitad de los encuestados considera que es importante dominar una lengua extranjera, en este caso el inglés y, considerando que apenas un trece por ciento cree que es importante hacer uso de las TIC como recurso en clase, sugeriría no solamente que es indispensable proyectar y reforzar la importancia de la adquisición de estas competencias dentro del master de educación secundaria, sino que también puede plantearse como una de las razones por las cuales el sesenta y uno por ciento de los encuestados percibe que no tiene buen desempeño profesional como docente.

Llama la atención que un porcentaje bastante significativo, $31 \%$, considera muy importante saber resolver los conflictos que se presentan dentro del aula; sin embargo, al cuestionarles sobre la aplicación de estrategias para el trabajo colaborativo en el aula, un $44.5 \%$ consideró que no es importante. Esto dice mucho de la formación y competencias que se desarrollan, adquiéranse o no, dentro del master de educación secundaria. ¿Cómo planteamos la resolución de conflictos en el aula sin darle importancia al trabajo colaborativo dentro de ésta? Es interesante encontrar que aquellos que provienen de las áreas de humanidades se sienten más competentes para ejercer la enseñanza que los que egresan de ingeniería. Esto quizás pueda deberse a que los de humanidades desarrollan más habilidades de comunicación que los de ingeniería; lo que sugiere que es preciso reforzarlo, sobre todo en lo que respecta a la teoría y la práctica de la enseñanza.

Un estudio que podría ser interesante, sería un posible seguimiento a los egresados que trabajan en la docencia 
y preguntarles qué competencias han tenido que adquirir por su cuenta o cuáles han echado en falta a la hora de ejercer como docentes; y quizás, por otro lado, también sería interesante realizar un seguimiento al $61 \%$ de los egresados que no trabaja como docente y preguntarles sobre las razones por las que no trabajan en la docencia, y si consideran que las competencias para las que habilita el master de educación secundaria, son o no las que les piden a la hora de solicitar un puesto de trabajo para laborar como docentes, o si consideran que hay competencias que les facilitarían acceder a un trabajo de docente. Sin descartar que es posible que una parte, aunque seguramente pequeña, no ejerza como docente por decisión propia.

\section{Referencias}

Aparicio, J. J., \& Moneo, M. R. (2016). La calidad de la educación como determinante del progreso de los países y la calidad del profesorado como determinante de la calidad de la educación. Tarbiya, revista de Investigación e Innovación Educativa, (44), 41-66.

De Juanas Oliva, Á. y Beltrán Llera, J.A. (2014). Valoraciones de los estudiantes de ciencias de la educación sobre la calidad de la docencia universitaria. Educación $\quad X X 1, \quad 17 \quad$ (1), 57-82. doi: 10.5944/educxx1.17.1.10705.

European Union (2009). Conclusiones del Consejo, de 12 de mayo de 2009, sobre un marco estratégico para la cooperación europea en el ámbito de la educación y la formación (ET 2020) [Diario Oficial C 119 de 28.5.2009].

European Union (2009). Council Conclusions of 26 November 2009 on the professional development of teachers and school leaders (Official Journal 2009/C 302/04, 12.12.2009).

European Union (2014). Conclusiones del Consejo, de 20 de mayo de 2014, sobre formación eficaz de los docentes (1) (2014/C 183/05) Diario Oficial de la Unión Europea 14.6.2014

Galaz, A. (2015). Fracturas de la identidad en la formación por competencias de los futuros profesores: Análisis de una experiencia. Revista de Pedagogía, 35, 97-98.

George, D. y Mallery, P. (2003). SPSS for Windows step by step: A simple guide and reference. 11.0 update $\left(4^{\mathrm{a}}\right.$ ed.). Boston, MA: Allyn \& Bacon.

Gliem, J. y Gliem, R. (2003). Calculating, Interpreting, and Reporting Cronbach's Alpha Reliability Coefficient for Likert-Type Scales. Conference in Adult, Continuing and Community Education. Midwest Research to Practice. The Ohio State University, Columbus.

Hagger, H. \& McIntyre, D. (2006). Learning teaching from teachers. Realizing the potential of school-based teacher education. Maidenhead: Open University Press.

Kelly, M. \& Grenfell, M. (2004). European Profile for Language Teacher Education. A Frame of Reference. University of Southampton, UK

Mir Pozo, M. L., \& Ferrer Ribot, M. (2014). Aproximación a la situación actual de la formación del profesorado de educación infantil. Revista electrónica interuniversitaria de formación del profesorado, 17(2), 255.

OCDE (2009). Creating Effective Teaching and Learning Environments. First Results from TALIS. Paris: OECD OCDE (2014). Resultados de TALIS 2013. An International Perspective on Teaching and Learning. París: OCDE.

Sijtsma, K. (2009). On the use, the misuse and the very limited of the Cronbach's alpha. Psychometrika, 74(1) 107-120.

Tribó, G. (2008). Un nuevo perfil profesional de los profesores de secundaria [Monografías en internet]. Madrid (España). 